Research Article

\title{
Some Difference Inequalities for Iterated Sums with Applications
}

\author{
Wu-Sheng Wang ${ }^{1}$ and Shanhe $\mathrm{Wu}^{2}$ \\ ${ }^{1}$ School of Mathematics and Statistics, Hechi University, Yizhou, Guangxi 546300, China \\ ${ }^{2}$ Department of Mathematics and Computer Science, Longyan University, Longyan, Fujian 364012, China
}

Correspondence should be addressed to Shanhe Wu; shanhewu@gmail.com

Received 4 September 2013; Revised 13 November 2013; Accepted 28 November 2013

Academic Editor: Jaume Giné

Copyright (c) 2013 W.-S. Wang and S. Wu. This is an open access article distributed under the Creative Commons Attribution License, which permits unrestricted use, distribution, and reproduction in any medium, provided the original work is properly cited.

The main objective of this paper is to establish two new nonlinear sum-difference inequalities with multiple iterated sums. Under several practical assumptions, the inequalities are solved through rigorous analysis, and explicit bounds for the unknown functions are given clearly. These new inequalities can be used as handy tools in the study of the estimation of solutions of difference equations.

\section{Introduction}

One of the best known and widely used inequalities in the study of nonlinear differential equations is Gronwall-Bellman inequality $[1,2]$, which can be stated as follows: if $u$ and $f$ are nonnegative continuous functions on an interval $[a, b]$ satisfying

$$
u(t) \leq c+\int_{a}^{t} f(s) u(s) d s, \quad t \in[a, b],
$$

for some constant $c \geq 0$, then

$$
u(t) \leq c \exp \left(\int_{a}^{t} f(s) d s\right), \quad t \in[a, b] .
$$

It has become one of the very few classic and most influential results in the theory and applications of inequalities. Because of its fundamental importance, over the years, many generalizations and analogous results of (2) have been established, such as [3-14].
Among these references, Bainov and Simeonov [4, P. 107] considered the following interesting Gronwall-type inequality:

$$
\begin{aligned}
& u(t) \\
& \leq a(t)+\sum_{i=1}^{n} \int_{\alpha}^{t} f_{1}\left(t, t_{1}\right) \\
& \quad \times\left(\int_{\alpha}^{t_{1}} f_{2}\left(t_{1}, t_{2}\right) \cdots\left(\int_{\alpha}^{t_{i-1}} f_{i}\left(t_{i-1}, t_{i}\right) u\left(t_{i}\right) d t_{i}\right) \cdots\right) d t_{1} .
\end{aligned}
$$

Kim [8] considered analogous Gronwall-type integral inequalities involving iterated integrals,

$$
\begin{aligned}
& u(t) \\
& \leq a+b(t)\left(\int_{\alpha}^{t} f_{1}\left(t_{1}\right) u\left(t_{1}\right) \log u\left(t_{1}\right) d t_{1}\right. \\
& +\sum_{i=2}^{n} \int_{\alpha}^{t} g_{1}\left(t_{1}\right)\left(\int_{\alpha}^{t_{1}} g_{2}\left(t_{2}\right)\right. \\
& \times\left(\cdots \left(\int_{\alpha}^{t_{i-2}} g_{i-1}\left(t_{i-1}\right)\right.\right.
\end{aligned}
$$




$$
\begin{gathered}
\times\left(\int_{\alpha}^{t_{i-1}} f_{i}\left(t_{i}\right) u\left(t_{i}\right)\right. \\
\left.\left.\times \log \left(u\left(t_{i}\right)\right) d t_{i}\right) d t_{i-1}\right) \\
\left.\left.\ldots) d t_{2}\right) d t_{1}\right)
\end{gathered}
$$

In 2011, Abdeldaim and Yakout [12] studied some new integral inequalities of Gronwall-Bellman-Pachpatte type such as

$$
\begin{gathered}
u(t) \\
\leq u_{0}+\int_{0}^{t} f(s) u(s) \\
\times\left[u(s)+\int_{0}^{s} h(\tau)[u(\tau)\right. \\
\left.\qquad \begin{array}{l}
\tau(t) \leq \\
u
\end{array} \quad+\int_{0}^{t}[f(\xi) u(\xi) d \xi] d \tau\right] d s, \\
+\int_{0}^{t} f(s) u(s)\left[u(s)+\int_{0}^{s} g(\tau) u(\tau) d \tau\right] d s .
\end{gathered}
$$

Along with the development of the theory of integral inequalities and the theory of difference equations, more and more attentions are paid to discrete versions of Gronwalltype inequalities; for detailed information, please refer to the literatures [15-35]. For instance, Pachpatte [19] considered the following discrete inequality:

$$
\begin{aligned}
u(n) \leq & u_{0}+\sum_{s=n_{0}}^{n-1} f(s) u(s)+\sum_{s=n_{0}}^{n-1} g(s) \\
& \times\left(\sum_{t=n_{0}}^{s-1} h(t)\left(\sum_{\tau=n_{0}}^{t-1} k(\tau) u^{p}(\tau)\right)\right) .
\end{aligned}
$$

In 2006, Cheung and Ren [24] studied

$$
\begin{aligned}
u^{p}(m, n) \leq & c+\sum_{s=m_{0}}^{m-1} \sum_{t=n_{0}}^{n-1} a(s, t) u^{q}(s, t) \\
& +\sum_{s=m_{0}}^{m-1} \sum_{t=n_{0}}^{n-1} b(s, t) u^{q}(s, t) w(u(s, t)) .
\end{aligned}
$$

Later, Zheng et al. [31] discussed the following discrete inequality:

$$
u(n) \leq a(n)+\sum_{i=1}^{k} \sum_{s=0}^{n-1} f_{i}(n, s) w_{i}(u(s))
$$

In 2012, Zhou et al. [33] studied the following inequalities:

$$
\begin{aligned}
u(n) \leq & a(n)+\sum_{s=n_{0}}^{n-1} f_{1}(n, s) w(u(s)) \\
& +\sum_{s=n_{0}}^{n-1} f_{1}(n, s) w(u(s)) \sum_{\tau=n_{0}}^{s-1} f_{2}(s, \tau) w(u(\tau)) \\
& +\sum_{s=n_{0}}^{n-1} f_{1}(n, s) w(u(s)) \\
& \times \sum_{\tau=n_{0}}^{s-1} f_{2}(s, \tau) \sum_{\xi=n_{0}}^{\tau-1} f_{3}(\tau, \xi) w(u(\xi)) \\
u(n) \leq & a(n)+\sum_{s=n_{0}}^{n-1} f_{1}(n, s) w_{1}(u(s)) \\
& +\sum_{\tau=n_{0}}^{n-1} f_{2}(s, \tau) w_{2}(u(\tau)) \sum_{\xi=n_{0}}^{\tau-1} f_{3}(\tau, \xi) w_{3}(u(\xi)) \\
& +\sum_{s=n_{0}}^{n-1} f_{1}(n, s) w_{1}(u(s)) \\
& \\
& \\
& \\
& \\
&
\end{aligned}
$$

However, the above results are not applicable to some certain inequalities with multiple iterated sums. Hence, it is desirable to consider more general difference inequalities of these extended types. They can be used in the study of certain classes of difference equations or applied in many practical engineering problems.

Motivated by the results given in $[7,8,12,19,24,25$, 29,33 ], in this paper we discuss the following two types of inequalities:

$$
\begin{aligned}
u(n) \leq & a(n)+\sum_{t_{1}=n_{0}}^{n-1} f_{1}\left(n, t_{1}\right) \\
& \times\left(\sum_{t_{2}=n_{0}}^{t_{1}-1} f_{2}\left(t_{1}, t_{2}\right) \cdots\left(\sum_{t_{k}=n_{0}}^{t_{k-1}} f_{k}\left(t_{k-1}, t_{k}\right) u^{p}\left(t_{k}\right)\right) \cdots\right),
\end{aligned}
$$


$u(n) \leq a(n)$

$$
\begin{array}{r}
+c(n)\left[\sum_{t_{1}=n_{0}}^{n-1} f_{1}\left(n, t_{1}\right) g\left(u\left(t_{1}\right)\right)+\sum_{i=2}^{k} \sum_{t_{1}=n_{0}}^{n-1} f_{1}\left(n, t_{1}\right)\right. \\
\times\left(\sum_{t_{2}=n_{0}}^{t_{1}-1} f_{2}\left(t_{1}, t_{2}\right) \cdots \times\left(\sum_{t_{i}=n_{0}}^{t_{i-1}} f_{i}\left(t_{i-1}, t_{i}\right)\right.\right. \\
\left.\left.\left.\times g\left(u\left(t_{i}\right)\right)\right) \cdots\right)\right] .
\end{array}
$$

All the assumptions on (10) and (11) are given in the next sections. The inequalities (10) and (11) consist of multiple iterated sums. Under several practical assumptions, the inequalities are solved through rigorous analysis, and explicit bounds for the unknown functions are given clearly. Further, the derived results are applied to study the estimation of solutions of difference equations.

\section{Main Results}

Throughout this paper, let $\mathbb{N}_{n_{0}}:=\left\{n_{0}, n_{0}+1, n_{0}+2, \ldots\right\}$ and $\mathbb{N}_{n_{0}}^{b}:=\left\{n_{0}, n_{0}+1, n_{0}+2, \ldots, n_{0}+n=b\right\}\left(n_{0} \in \mathbb{N}_{0}, n, b \in\right.$ $\mathbb{N})$. For function $u(n)$, its difference is defined by $\Delta u(n)=$ $u(n+1)-u(n)$. Obviously, the linear difference equation $\Delta u(n)=f(n)$ with the initial condition $u\left(n_{0}\right)=0$ has the solution $u(n)=\sum_{s=n_{0}}^{n-1} f(s)$. For convenience, in the sequel we complementarily define that $\sum_{s=n_{0}}^{n_{0}-1} f(s)=0$.

Lemma 1. Let $u(n), a(n)$ and $c(n)$ be real-valued nonnegative functions defined on $\mathbb{N}_{0}$ and satisfy the inequality

$$
\Delta u(n) \leq a(n) u(n)+c(n), \quad \forall n \in \mathbb{N}_{n_{0}},
$$

where $u_{n_{0}}$ is a nonnegative constant. Then,

$$
\begin{aligned}
u(n) \leq\left(u_{n_{0}}\right. & \left.+\sum_{s=n_{0}}^{n-1} c(s) \prod_{t=n_{0}}^{s}(1+a(t))^{-1}\right) \\
& \times \prod_{s=n_{0}}^{n-1}(1+a(s)), \quad \forall n \in \mathbb{N}_{n_{0}} .
\end{aligned}
$$

Proof. From (12), we have

$$
u(n+1)-(1+a(n)) u(n) \leq c(n), \quad \forall n \in \mathbb{N}_{n_{0}} .
$$

Multiplying by $\prod_{s=n_{0}}^{n-1}(1+a(s))^{-1}$ on both sides of the above inequality (14) and summing up both sides from $n_{0}$ to $n-1$, we obtain

$$
\begin{aligned}
u(n) & \prod_{s=n_{0}}^{n-1}(1+a(s))^{-1}-u_{n_{0}} \\
\leq & \sum_{s=n_{0}}^{n-1} c(s) \prod_{t=n_{0}}^{s}(1+a(t))^{-1}, \quad \forall n \in \mathbb{N}_{n_{0}} .
\end{aligned}
$$

From (15), we obtain the desired estimate (13).
Theorem 2. Let $u(n)$ and $a(n)$ be nonnegative functions defined on $\mathbb{N}_{n_{0}}$ with a(n) nondecreasing on $\mathbb{N}_{n_{0}}$. Moreover, let $f_{i}(n, s), i=1,2, \ldots, k$, be nonnegative functions for $n_{0} \leq s \leq$ $n\left(n_{0}, n, s \in \mathbb{N}_{n_{0}}\right)$ and nondecreasing in $n$ for fixed $s \in \mathbb{N}_{n_{0}}$. If $p \geq 0$ and $p$ is not equal to 1 , then the discrete inequality (10) gives

$$
u(n) \leq V_{1}(n, n), \quad \forall n \in \mathbb{N}_{n_{0}}^{b_{1}},
$$

where $V_{1}(n, n)$ can be successively determined from the formulas

$$
\begin{aligned}
& V_{k}(M, n) \\
& =\exp \left(W _ { 1 } ^ { - 1 } \left(W_{1}\left(\ln (a(M))+\sum_{s=n_{0}}^{M-1}\left(\sum_{i=1}^{k-1} f_{i}(M, s)\right)\right)\right.\right. \\
& \left.\left.+\sum_{s=n_{0}}^{n-1} f_{k}(M, s)\right)\right),
\end{aligned}
$$

$V_{j}(M, n)$

$$
\begin{aligned}
\leq & \left(a(M)+\sum_{s=n_{0}}^{n-1} f_{j}(M, s) V_{j+1}(M, s)\right. \\
& \left.\times \prod_{t=n_{0}}^{s}\left(1+\left(\sum_{i=1}^{j-1} f_{i}(M, t)-f_{j}(M, t)\right)\right)^{-1}\right) \\
& \times \prod_{s=n_{0}}^{n-1}\left(1+\left(\sum_{i=1}^{j-1} f_{i}(M, s)-f_{j}(M, s)\right)\right)=V_{j}(M, n),
\end{aligned}
$$

$$
\begin{aligned}
& \text { for } j=k-1, \ldots, 2,1, n \in \mathbb{N}_{n_{0}}^{M}, \\
& \qquad W_{1}(x)=\int_{x_{0}}^{x} \frac{d s}{\exp ((p-1) s)}, \quad x_{0}>0,
\end{aligned}
$$

where $W_{1}^{-1}$ is the inverse functions of $W_{1}, M \in \mathbb{N}_{n_{0}}, M \leq b_{1}$ is chosen arbitrarily, and $b_{1}$ is the largest natural number such that

$$
\begin{gathered}
W_{1}\left(\ln \left(a\left(b_{1}\right)\right)+\sum_{s=n_{0}}^{b_{1}-1}\left(\sum_{i=1}^{k-1} f_{i}\left(b_{1}, s\right)\right)\right) \\
\quad+\sum_{s=n_{0}}^{b_{1}-1} f_{k}\left(b_{1}, s\right) \in \operatorname{Dom}\left(W_{1}^{-1}\right) .
\end{gathered}
$$

Remark 3. Firstly, from (17) and (18), we obtain $V_{1}(M, n)$; then let $M=n$, and we get $V_{1}(n, n)$ since $M$ is chosen arbitrarily.

Remark 4. We can obtain $b_{1}$ using MATLAB program: firstly let $b_{1}=n_{0}$, when $W_{1}\left(\ln \left(a\left(b_{1}\right)\right)+\sum_{s=n_{0}}^{b_{1}-1}\left(\sum_{i=1}^{k-1} f_{i}\left(b_{1}, s\right)\right)\right)+$ 
$\sum_{s=n_{0}}^{b_{1}-1} f_{k}\left(b_{1}, s\right)<W_{1}(\infty)$; let $b_{1}=b_{1}+1$, when $W_{1}\left(\ln \left(a\left(b_{1}\right)\right)+\right.$ $\left.\sum_{s=n_{0}}^{b_{1}-1}\left(\sum_{i=1}^{k-1} f_{i}\left(b_{1}, s\right)\right)\right)+\sum_{s=n_{0}}^{b_{1}-1} f_{k}\left(b_{1}, s\right)<W_{1}(\infty) ;$ let $b_{1}=b_{1}+1$, and so on until $W_{1}\left(\ln \left(a\left(b_{1}\right)\right)+\right.$ $\left.\sum_{s=n_{0}}^{b_{1}-1}\left(\sum_{i=1}^{k-1} f_{i}\left(b_{1}, s\right)\right)\right)+\sum_{s=n_{0}}^{b_{1}-1} f_{k}\left(b_{1}, s\right) \geq W_{1}(\infty)$. If $W_{1}\left(\ln \left(a\left(b_{1}\right)\right)+\sum_{s=n_{0}}^{b_{1}-1}\left(\sum_{i=1}^{k-1} f_{i}\left(b_{1}, s\right)\right)\right)+\sum_{s=n_{0}}^{b_{1}-1} f_{k}\left(b_{1}, s\right)<$ $W_{1}(\infty)$, for all $b_{1} \in \mathbb{N}_{n_{0}}$, then $b_{1}=\infty$.

Proof. Fix $M \in \mathbb{N}_{n_{0}}^{b_{1}}$, where $M$ is chosen arbitrarily and $b_{1}$ is defined by (20). For $n \in \mathbb{N}_{n_{0}}^{M}$, from (10) we have

$$
\begin{aligned}
u(n) \leq & a(M)+\sum_{t_{1}=n_{0}}^{n-1} f_{1}\left(M, t_{1}\right) \\
& \times\left(\sum_{t_{2}=n_{0}}^{t_{1}-1} f_{2}\left(M, t_{2}\right) \cdots\left(\sum_{t_{k}=n_{0}}^{t_{k-1}} f_{k}\left(M, t_{k}\right) u^{p}\left(t_{k}\right)\right) \cdots\right) .
\end{aligned}
$$

Now we introduce the functions

$$
\begin{aligned}
v_{1}(n)= & a(M)+\sum_{t_{1}=n_{0}}^{n-1} f_{1}\left(M, t_{1}\right) \\
& \times\left(\sum_{t_{2}=n_{0}}^{t_{1}-1} f_{2}\left(M, t_{2}\right) \cdots\left(\sum_{t_{k}=n_{0}}^{t_{k-1}} f_{k}\left(M, t_{k}\right) u^{p}\left(t_{k}\right)\right) \cdots\right),
\end{aligned}
$$

$$
\begin{aligned}
v_{j}(n)= & v_{j-1}(n)+\sum_{t_{j}=n_{0}}^{n-1} f_{j}\left(M, t_{j}\right) \\
& \times\left(\sum_{t_{j+1}=n_{0}}^{t_{j}-1} f_{j+1}\left(M, t_{j+1}\right) \cdots\right. \\
& \left.\times\left(\sum_{t_{k}=n_{0}}^{t_{k-1}} f_{k}\left(M, t_{k}\right) u^{p}\left(t_{k}\right)\right) \cdots\right) .
\end{aligned}
$$

For $n \in \mathbb{N}_{n_{0}}^{M}$ and $j=2,3, \ldots, k$, then $v_{j}, j=1,2, \ldots, k$, are all positive and nondecreasing functions on $\mathbb{N}_{n_{0}}^{M}$ with $v_{j}\left(n_{0}\right)=$ $a(M), j=1,2, \ldots, k$, and the inequalities (22) and (23) imply that

$$
u(n) \leq v_{1}(n) \leq v_{2}(n) \leq \cdots \leq v_{k}(n), \quad \forall n \in \mathbb{N}_{n_{0}}^{M}
$$

From (22), we observe that

$$
\begin{aligned}
\Delta v_{1}(n)= & v_{1}(n+1)-v_{1}(n) \\
= & f_{1}(M, n) \\
& \times\left(\sum_{t_{2}=n_{0}}^{n-1} f_{2}\left(M, t_{2}\right) \cdots\left(\sum_{t_{k}=n_{0}}^{t_{k-1}} f_{k}\left(M, t_{k}\right) u^{p}\left(t_{k}\right)\right) \cdots\right) \\
\leq & f_{1}(M, n)\left(v_{2}(n)-v_{1}(n)\right) \\
= & -f_{1}(M, n) v_{1}(n)+f_{1}(M, n) v_{2}(n), \quad \forall n \in \mathbb{N}_{n_{0}}^{M} .
\end{aligned}
$$

We claim that

$$
\begin{gathered}
\Delta v_{j}(n) \leq\left(\sum_{i=1}^{j-1} f_{i}(M, n)-f_{j}(M, n)\right) v_{j}(n) \\
+f_{j}(M, n) v_{j+1}(n), \\
\Delta v_{k}(n) \leq\left(\sum_{i=1}^{k-1} f_{i}(M, n)\right) v_{k}(n)+f_{k}(M, n) v_{k}^{p}(n),
\end{gathered}
$$

for $n \in \mathbb{N}_{n_{0}}^{M}, j=2,3, \ldots, k-1$.

Now we prove (26) and (27) by induction. Obviously, (26) is true for $j=1$ by (26). We make the inductive assumption that (26) is true for $j-1$. By the inductive assumption and (24), from (23) we obtain

$$
\begin{aligned}
\Delta v_{j}(n) \leq & \Delta v_{j-1}(n)+f_{j}(M, n) \\
& \times\left(\sum_{t_{j+1}=n_{0}}^{t_{j}-1} f_{j+1}\left(M, t_{j+1}\right) \ldots\right. \\
& \left.\times\left(\sum_{t_{k}=n_{0}}^{t_{k-1}} f_{k}\left(M, t_{k}\right) u^{p}\left(t_{k}\right)\right) \ldots\right) \\
\leq & \left(\sum_{i=1}^{j-2} f_{i}(M, n)-f_{j-1}(M, n)\right) v_{j-1}(n) \\
& +f_{j-1}(M, n) v_{j}(n)+f_{j}(M, n)\left(v_{j+1}(n)-v_{j}(n)\right) \\
\leq & \left(\sum_{i=1}^{j-2} f_{i}(M, n)\right) v_{j}(n)+f_{j-1}(M, n) v_{j}(n) \\
& +f_{j}(M, n) v_{j+1}(n)-f_{j}(M, n) v_{j}(n) \\
= & \left(\sum_{i=1}^{j-1} f_{i}(M, n)-f_{j}(M, n)\right) v_{j}(n) \\
& +f_{j}(M, n) v_{j+1}(n), \quad \forall n \in \mathbb{N}_{n_{0}}^{M} .
\end{aligned}
$$


It actually proves (26) by induction. From (23) and (26), we have

$$
\begin{aligned}
& \Delta v_{k}(n)= \Delta v_{k-1}(n)+f_{k}(M, n) u^{p}(n) \\
& \leq\left(\sum_{i=1}^{k-2} f_{i}(M, n)-f_{k-1}(M, n)\right) v_{k-1}(n) \\
&+f_{k-1}(M, n) v_{k}(n)+f_{k}(M, n) v_{k}^{p}(n) \\
& \leq\left(\sum_{i=1}^{k-1} f_{i}(M, n)\right) v_{k}(n)+f_{k}(M, n) v_{k}^{p}(n), \\
& \forall n \in \mathbb{N}_{n_{0}}^{M} .
\end{aligned}
$$

It proves (27). From (27), we have

$$
\begin{array}{r}
\frac{\Delta v_{k}(n)}{v_{k}(n)} \leq\left(\sum_{i=1}^{k-1} f_{i}(M, n)\right)+f_{k}(M, n) v_{k}^{p-1}(n), \\
\forall n \in \mathbb{N}_{n_{0}}^{M} .
\end{array}
$$

On the other hand, by the mean-value theorem for integrals, for arbitrarily given integers $n, n+1 \in \mathbb{N}_{n_{0}}^{M}$, there exists $\xi$ in the open interval $\left(v_{k}(n), v_{k}(n+1)\right)$ such that

$$
\begin{aligned}
\ln \left(v_{k}(n+1)\right)-\ln \left(v_{k}(n)\right)= & \int_{v_{k}(n)}^{v_{k}(n+1)} \frac{d s}{s} \\
=\frac{\Delta v_{k}(n)}{\xi} & \leq \frac{\Delta v_{k}(n)}{v_{k}(n)}, \\
& \forall n \in \mathbb{N}_{n_{0}}^{M} .
\end{aligned}
$$

By setting $n=s$ in (31) and substituting $s=n_{0}, n_{0}+1, n_{0}+$ $2, \ldots, n-1$, successively, we obtain

$$
\begin{aligned}
\ln \left(v_{k}(n)\right) \leq & \ln \left(v_{k}\left(n_{0}\right)\right)+\sum_{s=n_{0}}^{n-1}\left(\sum_{i=1}^{k-1} f_{i}(M, s)\right) \\
& +\sum_{s=n_{0}}^{n-1} f_{k}(M, s) v_{k}^{p-1}(s) \\
\leq & \ln \left(v_{k}\left(n_{0}\right)\right)+\sum_{s=n_{0}}^{M-1}\left(\sum_{i=1}^{k-1} f_{i}(M, s)\right) \\
& +\sum_{s=n_{0}}^{n-1} f_{k}(M, s) v_{k}^{p-1}(s), \quad \forall n \in \mathbb{N}_{n_{0}}^{M} .
\end{aligned}
$$

Let $w_{1}(n)$ denote the right-hand side of (32), which is a positive and nondecreasing function on $\mathbb{N}_{n_{0}}^{M}$ with

$$
w_{1}\left(n_{0}\right)=\ln \left(v_{k}\left(n_{0}\right)\right)+\sum_{s=n_{0}}^{M-1}\left(\sum_{i=1}^{k-1} f_{i}(M, s)\right) .
$$

Then, (32) is equivalent to

$$
v_{k}(n) \leq \exp \left(w_{1}(n)\right), \quad \forall n \in \mathbb{N}_{n_{0}}^{M} .
$$

By the definition of $w_{1}$, we obtain

$$
\begin{aligned}
& \Delta w_{1}(n)=f_{k}(M, n) v_{k}^{p-1}(n) \\
& \leq f_{k}(M, n) \exp \left((p-1) w_{1}(n)\right), \\
& \forall n \in \mathbb{N}_{n_{0}}^{M} .
\end{aligned}
$$

From (34) and (35), we get

$$
\frac{\Delta w_{1}(n)}{\exp \left((p-1) w_{1}(n)\right)} \leq f_{k}(M, n), \quad \forall n \in \mathbb{N}_{n_{0}}^{M} .
$$

Once again, performing the similar procedure from (30) to (32), (36) gives

$$
\begin{array}{r}
W_{1}\left(w_{1}(n)\right) \leq W_{1}\left(w_{1}\left(n_{0}\right)\right)+\sum_{s=n_{0}}^{n-1} f_{k}(M, s), \\
\forall n \in \mathbb{N}_{n_{0}}^{M},
\end{array}
$$

where $W_{1}$ is defined by (19). By combining (33), (34), and (37), we can obtain that

$$
\begin{aligned}
v_{k}(n) & \leq \exp \left(w_{1}(n)\right) \\
& \leq \exp \left(W_{1}^{-1}\left(W_{1}\left(w_{1}\left(n_{0}\right)\right)+\sum_{s=n_{0}}^{n-1} f_{k}(M, s)\right)\right) \\
& \leq \exp \left(W _ { 1 } ^ { - 1 } \left(W_{1}(\ln (a(M))\right.\right. \\
& \left.+\sum_{s=n_{0}}^{M-1}\left(\sum_{i=1}^{k-1} f_{i}(M, s)\right)\right) \\
& \left.\left.=V_{k}(M, n), \quad \forall n \in \sum_{s=n_{0}}^{n-1} f_{k}(M, s)\right)\right)
\end{aligned}
$$

where $V_{k}(M, n)$ is defined by (17). Applying Lemma 1 to (26) for $j=k-1, \ldots, 2,1$, we have

$$
\begin{aligned}
v_{j}(n) \leq & \left(a(M)+\sum_{s=n_{0}}^{n-1} f_{j}(M, s) V_{j+1}(M, s)\right. \\
& \left.\times \prod_{t=n_{0}}^{s}\left(1+\left(\sum_{i=1}^{j-1} f_{i}(M, t)-f_{j}(M, t)\right)\right)^{-1}\right) \\
& \times \prod_{s=n_{0}}^{n-1}\left(1+\left(\sum_{i=1}^{j-1} f_{i}(M, s)-f_{j}(M, s)\right)\right) \\
= & V_{j}(M, n), \quad \forall n \in \mathbb{N}_{n_{0}}^{M},
\end{aligned}
$$

where $V_{j}(M, n)$ is defined by (18). From (24) and (39), we have

$$
u(n) \leq v_{1}(n) \leq V_{1}(M, n), \quad \forall n \in \mathbb{N}_{n_{0}}^{M} .
$$


Since $M \in \mathbb{N}_{n_{0}}$ is arbitrary, from (40), we get the required estimate

$$
u(n) \leq V_{1}(n, n), \quad \forall n \in \mathbb{N}_{n_{0}}^{b_{1}},
$$

where $b_{1}$ is defined by (20). Theorem 2 is proved.

Theorem 5. Let $u(n), a(n)$, and $c(n)$ be nonnegative functions defined on $\mathbb{N}_{n_{0}}$ with $a(n)$ and $c(n)$ nondecreasing on $\mathbb{N}_{n_{0}}$, and let $f_{i}(n, s), i=1,2, \ldots, k$, be nonnegative functions for $n, s \in$ $\mathbb{N}_{n_{0}}, n_{0} \leq s \leq n$, which are nondecreasing in $n$ for fixed $s \in \mathbb{N}_{n_{0}}$. Suppose that $g(u)$ is a nondecreasing continuous function on $[0, \infty)$ with $g(u)>0$ for $u>0$. The inequality (11) implies that

$$
\begin{array}{r}
u(n) \leq G^{-1}\left(G(a(n))+c(n) \sum_{s=n_{0}}^{n-1} f_{1}(n, s) E(n, s)\right) \\
\forall n \in \mathbb{N}_{n_{0}}^{b_{2}},
\end{array}
$$

where $G^{-1}$ is the inverse function of $G$,

$$
G(u)=\int_{u_{0}}^{u} \frac{d s}{g(s)}, \quad u_{0}>0,
$$

$E(n, s)$

$$
\begin{aligned}
:=\left\{1+f_{2}(n, s)\left[1+f_{3}(\right.\right. & n, s) \\
\times(\cdots & \left(1+f_{k-1}(n, s) \cdots\right. \\
& \left.\left.\left.\left.\times\left(1+f_{k}(n, s)\right)\right) \cdots\right)\right]\right\},
\end{aligned}
$$

and $b_{2}$ is the largest natural number such that

$$
G\left(a\left(b_{2}\right)\right)+c\left(b_{2}\right) \sum_{s=n_{0}}^{b_{2}-1} f_{1}\left(b_{2}, s\right) E\left(b_{2}, s\right) \in \operatorname{Dom}\left(G^{-1}\right) .
$$

Remark 6. We can obtain $b_{2}$ using Matlab program similar to Remark 4.

Proof. Let the function $a(n)$ be positive. Fix $M \in \mathbb{N}_{n_{0}}^{b_{2}}$, where $M$ is chosen arbitrarily and $b_{2}$ is defined by (45). For $n \in \mathbb{N}_{n_{0}}^{M}$, from (11) we have

$$
\begin{aligned}
& u(n) \\
& \leq a(M)+c(M)\left[\sum_{t_{1}=n_{0}}^{n-1} f_{1}\left(M, t_{1}\right) g\left(u\left(t_{1}\right)\right)\right. \\
& +\sum_{i=2}^{k} \sum_{t_{1}=n_{0}}^{n-1} f_{1}\left(M, t_{1}\right) \\
& \times\left(\sum_{t_{2}=n_{0}}^{t_{1}-1} f_{2}\left(M, t_{2}\right) \cdots\right. \\
& \\
& \left.\left.\quad \times\left(\sum_{t_{i}=n_{0}}^{t_{i-1}} f_{i}\left(M, t_{i}\right) g\left(u\left(t_{i}\right)\right)\right) \ldots\right)\right] .
\end{aligned}
$$

We denote the right-hand side of (46) by $y(n)$ for $n \epsilon$ $\mathbb{N}_{n_{0}}^{M}$. Then $y\left(n_{0}\right)=a(M)$, the function $y(n)$ is positive and nondecreasing in $n \in \mathbb{N}_{n_{0}}^{M}, u(n) \leq y(n)$, and

$\Delta y(n)$

$$
\begin{aligned}
&=c(M)\left[f_{1}(M, n) g(u(n))+f_{1}(M, n)\right. \\
& \times\left(\sum_{t_{2}=n_{0}}^{n-1} f_{2}\left(M, t_{2}\right) g\left(u\left(t_{2}\right)\right)\right)+\sum_{i=3}^{k} f_{1}(M, n) \\
& \times\left(\sum_{t_{2}=n_{0}}^{n-1} f_{2}\left(M, t_{2}\right) \ldots\right. \\
&\left.\left.\times\left(\sum_{t_{i}=n_{0}}^{t_{i-1}} f_{i}\left(M, t_{i}\right) g\left(u\left(t_{i}\right)\right)\right) \ldots\right)\right] \\
&=c(M) f_{1}((n)\left[g(u(n))+\sum_{t_{2}=n_{0}}^{n-1} f_{2}\left(M, t_{2}\right) g\left(u\left(t_{2}\right)\right)\right. \\
&+\sum_{i=3}^{k}\left(\sum_{t_{2}=n_{0}}^{n-1} f_{2}\left(M, t_{2}\right) \cdots\right. \\
&\left.\left.\times\left(\sum_{t_{i}=n_{0}}^{t_{i-1}} f_{i}\left(M, t_{i}\right) g\left(u\left(t_{i}\right)\right)\right) \ldots\right)\right]
\end{aligned}
$$

Define a function $y_{1}(n)$ by

$$
\begin{aligned}
y_{1}(n)=\sum_{t_{2}=n_{0}}^{n-1} f_{2}\left(M, t_{2}\right) g\left(u\left(t_{2}\right)\right) & \\
& +\sum_{i=3}^{k}\left(\sum_{t_{2}=n_{0}}^{n-1} f_{2}\left(M, t_{2}\right) \cdots\right. \\
& \left.\times\left(\sum_{t_{i}=n_{0}}^{t_{i-1}} f_{i}\left(M, t_{i}\right) g\left(u\left(t_{i}\right)\right)\right) \cdots\right),
\end{aligned}
$$

for all $n \in \mathbb{N}_{n_{0}}^{M}$. From (47) and (48), we have

$$
\Delta y(n)=c(M) f_{1}(M, n)\left[g(u(n))+y_{1}(n)\right], \quad \forall n \in \mathbb{N}_{n_{0}}^{M} .
$$


From (48), we have

$\Delta y_{1}(n)$

$$
\begin{aligned}
f_{2}(M, n)\left[g(u(n))+\sum_{t_{3}=n_{0}}^{n-1} f_{3}\left(M, t_{3}\right) g\left(u\left(t_{3}\right)\right)\right. & \\
& +\sum_{i=4}^{k}\left(\sum_{t_{3}=n_{0}}^{n-1} f_{3}\left(M, t_{3}\right) \cdots\right. \\
& \left.\left.\times\left(\sum_{t_{i}=n_{0}}^{t_{i-1}} f_{i}\left(M, t_{i}\right) g\left(u\left(t_{i}\right)\right)\right) \cdots\right)\right], \\
& \forall n \in \mathbb{N}_{n_{0}}^{M} .
\end{aligned}
$$

From (50), we get

$$
\Delta y_{1}(n)=f_{2}(M, n)\left[g(u(n))+y_{2}(n)\right], \quad \forall n \in \mathbb{N}_{n_{0}}^{M},
$$

where

$y_{2}(n)$

$$
\begin{aligned}
= & \sum_{t_{3}=n_{0}}^{n-1} f_{3}\left(M, t_{3}\right) g\left(u\left(t_{3}\right)\right) \\
& +\sum_{i=4}^{k}\left(\sum_{t_{2}=n_{0}}^{n-1} f_{3}\left(M, t_{3}\right) \cdots\left(\sum_{t_{i}=n_{0}}^{t_{i-1}} f_{i}\left(M, t_{i}\right) g\left(u\left(t_{i}\right)\right)\right) \cdots\right),
\end{aligned}
$$

for all $n \in \mathbb{N}_{n_{0}}^{M}$.

Continuing in this way, we obtain

$$
\Delta y_{k-2}(n)=f_{k-1}(M, n)\left[g(u(n))+y_{k-1}(n)\right], \quad \forall n \in \mathbb{N}_{n_{0}}^{M},
$$

where

$$
y_{k-1}(n)=\sum_{t_{n}=n_{0}}^{n-1} f_{k}\left(M, t_{k}\right) g\left(u\left(t_{k}\right)\right), \quad \forall n \in \mathbb{N}_{n_{0}}^{M} .
$$

From (54) and the inequality $u(n) \leq y(n)$, we have

$$
\frac{\Delta y_{k-1}(n)}{g(y(n))} \leq f_{k}(M, n), \quad \forall n \in \mathbb{N}_{n_{0}}^{M} .
$$

We define the functions $\tilde{y}(s), \tilde{y}_{i}(s)(i=1,2 \ldots, k-1)$, which are nondecreasing and continuously differentiable on $\left[n_{0}, \infty\right)$ with $\tilde{y}(n)=y(n), \tilde{y}_{i}(n)=y_{i}(n)(i=1,2 \ldots, k-1)$ on $\mathbb{N}_{n_{0}}^{M}$.

On the other hand, by the formula of partial integration, we have

$$
\begin{aligned}
\int_{n}^{n+1} & \frac{\tilde{y}_{k-1}^{\prime}(s)}{g(\tilde{y}(s))} d s \\
= & \frac{y_{k-1}(n)}{g(y(n))} \\
& +\int_{n}^{n+1} \frac{\tilde{y}_{k-1}(s) g^{\prime}(\tilde{y}(s)) \tilde{y}^{\prime}(s)}{g^{2}(\tilde{y}(s))} d s, \quad \forall n \in \mathbb{N}_{n_{0}}^{M} .
\end{aligned}
$$

By the monotonicity of $g, y$, from (56) we have

$$
\int_{n}^{n+1} \frac{\tilde{y}_{k-1}^{\prime}(s)}{g(\tilde{y}(s))} d s \geq \frac{y_{k-1}(n)}{g(y(n))}, \quad \forall n \in \mathbb{N}_{n_{0}}^{M} .
$$

By the mean-value theorem for integrals, for arbitrarily given integers $n, n+1 \in \mathbb{N}_{n_{0}}^{M}$, there exists $\xi$ in the open interval $(n, n+1)$ such that

$$
\begin{aligned}
\int_{n}^{n+1} \frac{\tilde{y}_{k-1}^{\prime}(s)}{g(\tilde{y}(s))} d s & =\int_{n}^{n+1} \frac{d\left(\tilde{y}_{k-1}(s)\right)}{g(\tilde{y}(s))} \\
& =\frac{1}{g(y(\xi))} \int_{n}^{n+1} d\left(\tilde{y}_{k-1}(s)\right) \\
& \leq \frac{\Delta y_{k-1}(n)}{g(y(n))}, \quad \forall n \in \mathbb{N}_{n_{0}}^{M} .
\end{aligned}
$$

From (55), (57), and (58), we have

$$
\frac{y_{k-1}(n)}{g(y(n))} \leq \frac{\Delta y_{k-1}(n)}{g(y(n))} \leq f_{k}(M, n), \quad \forall n \in \mathbb{N}_{n_{0}}^{M}
$$

Next, from the inequalities (53) and (59), we have

$$
\frac{\Delta y_{k-2}(n)}{g(y(n))} \leq f_{k-1}(M, n)\left[1+f_{k}(M, n)\right], \quad \forall n \in \mathbb{N}_{n_{0}}^{M}
$$

Once again, applying the same procedure from (56) to (59) to the inequality (60), we get

$$
\begin{array}{r}
\frac{y_{k-2}(n)}{g(y(n))} \leq \frac{\Delta y_{k-2}(n)}{g(y(n))} \leq f_{k-1}(M, n)\left[1+f_{k}(M, n)\right] \\
\forall n \in \mathbb{N}_{n_{0}}^{M} .
\end{array}
$$

Proceeding in this way, we obtain

$$
\begin{aligned}
& \frac{y_{1}(n)}{g(y(n))} \\
& \leq \frac{\Delta y_{1}(n)}{g(y(n))} \leq f_{2}(M, n) \\
& \quad \times\left\{1+f_{3}(M, n)\right. \\
& \quad \times\left[1+f_{4}(M, n)\right. \\
& \quad \times\left(\cdots \left(1+f_{k-1}(M, n)\right.\right. \\
& \left.\left.\left.\left.\quad \times\left(1+f_{k}(M, n)\right)\right) \cdots\right)\right]\right\}, \\
& \quad \forall n \in \mathbb{N}_{n_{0}}^{M} .
\end{aligned}
$$


Using the inequalities (49) and (62), we have

$$
\begin{aligned}
& \frac{\Delta y(n)}{g(y(n))} \leq c(M) f_{1}(M, n) \\
& \times\left\{1+f_{2}(M, n)[1\right.+f_{3}(M, n) \\
& \times\left(\cdots \left(1+f_{k-1}(M, n)\right.\right. \\
&\left.\left.\left.\left.\times 1+f_{k}(M, n)\right) \cdots\right)\right]\right\} \\
&=c(M) f_{1}(M, n) E(M, n), \quad \forall n \in \mathbb{N}_{n_{0}}^{M},
\end{aligned}
$$

where $E(M, n)$ is defined by (44).

Once again, using the mean-value theorem for integrals, for arbitrarily given integers $n, n+1 \in \mathbb{N}_{n_{0}}^{M}$, there exists $\xi$ in the open interval $(y(n), y(n+1))$ such that

$$
\begin{aligned}
G(y(n+1))-G(y(n))= & \int_{y(n)}^{y(n+1)} \frac{d s}{g(s)} \\
= & \frac{\Delta y(n)}{g(\xi)} \leq \frac{\Delta y(n)}{g(y(n))}, \\
& \forall n \in \mathbb{N}_{n_{0}}^{M},
\end{aligned}
$$

where $G$ is defined by (43). Using (63), (64), and $y\left(n_{0}\right)=$ $a(M)$, we obtain

$$
\begin{aligned}
& u(n) \leq y(n) \leq G^{-1}( \\
&\left.+c(M) \sum_{s=n_{0}}^{n-1} f_{1}(M, s) E(M, s)\right), \\
& \forall n \in \mathbb{N}_{n_{0}}^{M} .
\end{aligned}
$$

In (65), let $n=M$; we have

$u(M)$

$$
\begin{array}{r}
\leq G^{-1}\left(G(a(M))+c(M) \sum_{s=n_{0}}^{M-1} f_{1}(M, s) E(M, s)\right), \\
\forall n \in \mathbb{N}_{n_{0}}^{M} .
\end{array}
$$

Due to the randomness of $T,(42)$ is achieved immediately from (66).

\section{Application}

In this section, we apply Theorem 5 to the following difference equation:

$$
\Delta x(n)=F\left(n, x(n), \sum_{s=n_{0}}^{n-1} z(s, x(s))\right), \quad \forall n \in \mathbb{N}_{n_{0}} .
$$

Corollary 7. Assume that $F$ is defined on $\mathbb{N}_{n_{0}} \times[0, \infty) \times[0, \infty)$, and there exist nonnegative functions $d_{i}(n), i=1,2$, such that

$$
\begin{gathered}
|F(n, x, y)| \leq d_{1}(n) g(|x|)+d_{1}(n) y, \\
|z(s, x)| \leq d_{2}(n) g(|x|),
\end{gathered}
$$

where the function $g$ is as in Theorem 5. If $x(n)$ is any solution of the problem (67), then

$$
|x(n)| \leq G^{-1}\left(G\left(\left|x\left(n_{0}\right)\right|\right)+\sum_{s=n_{0}}^{n-1} d_{1}(s) E(s)\right), \quad \forall n \in \mathbb{N}_{n_{0}}^{b_{3}},
$$

where the functions $G, G^{-1}$ are as in Theorem 5,

$$
E(n)=1+d_{1}(n)\left(1+d_{2}(n)\right),
$$

and $b_{3}$ is the largest natural number such that

$$
G\left(\left|x\left(n_{0}\right)\right|\right)+\sum_{s=n_{0}}^{b_{3}-1} d_{1}(s) E(s) \in \operatorname{Dom}\left(G^{-1}\right)
$$

Proof. The difference equation (67) is equivalent to

$$
\begin{array}{r}
x(n)=x\left(n_{0}\right)+\sum_{s=n_{0}}^{n-1} F\left(s, x(s), \sum_{t=n_{0}}^{s-1} z(t, x(t))\right), \\
\forall n \in \mathbb{N}_{n_{0}} .
\end{array}
$$

Using (68), from (72), we have

$$
\begin{aligned}
|x(n)| \leq & \left|x\left(n_{0}\right)\right|+\sum_{s=n_{0}}^{n-1} d_{1}(s) g(|x(s)|) \\
& +\sum_{s=n_{0}}^{n-1} d_{1}(s)\left(\sum_{t=n_{0}}^{s-1} d_{2}(t) g(|x(s)|)\right),
\end{aligned}
$$

$\forall n \in \mathbb{N}_{n_{0}}$.

Notice that, by the assumption, all functions in (73) satisfy the conditions of Theorem 5. Applying Theorem 5 to the inequality (73), (69) is immediately derived. This completes the proof of Corollary 7.

\section{Conflict of Interests}

The authors declare that they have no competing interests.

\section{Acknowledgments}

This research was supported, in part, by the National Natural Science Foundation of China under Grant 11161018 by the Guangxi Natural Science Foundation of China under Grant 2012GXNSFAA053009, and by the Natural Science Foundation of Fujian province of China under Grant 2012J01014. The authors are grateful to the anonymous referees for their careful comments and valuable suggestions on this paper. 


\section{References}

[1] T. H. Gronwall, "Note on the derivatives with respect to a parameter of the solutions of a system of differential equations," Annals of Mathematics, vol. 20, no. 4, pp. 292-296, 1919.

[2] R. Bellman, "The stability of solutions of linear differential equations," Duke Mathematical Journal, vol. 10, pp. 643-647, 1943.

[3] I. Bihari, "A generalization of a lemma of Bellman and its application to uniqueness problems of differential equations," Acta Mathematica Academiae Scientiarum Hungaricae, vol. 7, pp. 81-94, 1956.

[4] D. Baĭnov and P. Simeonov, Integral Inequalities and Applications, vol. 57 of Mathematics and Its Applications, Kluwer Academic Publishers, Dordrecht, The Netherlands, 1992.

[5] B. G. Pachpatte, Inequalities for Differential and Integral Equations, vol. 197 of Mathematics in Science and Engineering, Academic Press, San Diego, Calif, USA, 1998.

[6] O. Lipovan, "A retarded Gronwall-like inequality and its applications," Journal of Mathematical Analysis and Applications, vol. 252, no. 1, pp. 389-401, 2000.

[7] W. Zhang and S. Deng, "Projected Gronwall-Bellman's inequality for integrable functions," Mathematical and Computer Modelling, vol. 34, no. 3-4, pp. 393-402, 2001.

[8] B.-I. Kim, "On some Gronwall type inequalities for a system integral equation," Bulletin of the Korean Mathematical Society, vol. 42, no. 4, pp. 789-805, 2005.

[9] B. G. Pachpatte, "On certain nonlinear integral inequalities involving iterated integrals," Tamkang Journal of Mathematics, vol. 37, no. 3, pp. 261-271, 2006.

[10] W.-S. Cheung, "Some new nonlinear inequalities and applications to boundary value problems," Nonlinear Analysis: Theory, Methods \& Applications, vol. 64, no. 9, pp. 2112-2128, 2006.

[11] W.-S. Wang, "A generalized retarded Gronwall-like inequality in two variables and applications to BVP," Applied Mathematics and Computation, vol. 191, no. 1, pp. 144-154, 2007.

[12] A. Abdeldaim and M. Yakout, "On some new integral inequalities of Gronwall-Bellman-Pachpatte type," Applied Mathematics and Computation, vol. 217, no. 20, pp. 7887-7899, 2011.

[13] Z. Guo, X. Zhou, and W.-S. Wang, "Interval oscillation criteria of second order mixed nonlinear impulsive differential equations with delay," Abstract and Applied Analysis, vol. 2012, Article ID 351709, 23 pages, 2012.

[14] W. S. Wang, D. Huang, and X. Li, "Generalized retarded nonlinear integral inequalities involving iterated integrals and an application," Journal of Inequalities and Applications, vol. 2013, article 376, 2013.

[15] T. E. Hull and W. A. J. Luxemburg, "Numerical methods and existence theorems for ordinary differential equations," Numerische Mathematik, vol. 2, no. 1, pp. 30-41, 1960.

[16] D. Willett and J. S. W. Wong, "On the discrete analogues of some generalizations of Gronwall's inequality," Monatshefte für Mathematik, vol. 69, pp. 362-367, 1965.

[17] S. Sugiyama, "On the stability problems of difference equations," Bulletin of Science and Engineering Research Laboratory. Waseda University, vol. 45, pp. 140-144, 1969.

[18] B. G. Pachpatte and S. G. Deo, "Stability of discrete-time systems with retarded argument," Utilitas Mathematica, vol. 4, pp. 15-33, 1973.

[19] B. G. Pachpatte, "On discrete inequalities related to Gronwall's inequality," Proceedings of the Indian Academy of Sciences A, vol. 85 , no. 1 , pp. $26-40,1977$.
[20] B. G. Pachpatte, "Finite difference inequalities and discrete time control systems," Indian Journal of Pure and Applied Mathematics, vol. 9, no. 12, pp. 1282-1290, 1978.

[21] E. Yang, "A new nonlinear discrete inequality and its application," Annals of Differential Equations, vol. 17, no. 3, pp. 261-267, 2001.

[22] B. G. Pachpatte, "On some fundamental integral inequalities and their discrete analogues," Journal of Inequalities in Pure and Applied Mathematics, vol. 2, no. 2, article 15, 2001.

[23] F. W. Meng and W. N. Li, "On some new nonlinear discrete inequalities and their applications," Journal of Computational and Applied Mathematics, vol. 158, no. 2, pp. 407-417, 2003.

[24] W.-S. Cheung and J. Ren, "Discrete non-linear inequalities and applications to boundary value problems," Journal of Mathematical Analysis and Applications, vol. 319, no. 2, pp. 708-724, 2006.

[25] B. G. Pachpatte, Integral and Finite Difference Inequalities and Applications, vol. 205 of North-Holland Mathematics Studies, Elsevier Science, Amsterdam, The Netherlands, 2006.

[26] W. Sheng and W. N. Li, "Bounds on certain nonlinear discrete inequalities," Journal of Mathematical Inequalities, vol. 2, no. 2, pp. 279-286, 2008.

[27] Q.-H. Ma and W.-S. Cheung, "Some new nonlinear difference inequalities and their applications," Journal of Computational and Applied Mathematics, vol. 202, no. 2, pp. 339-351, 2007.

[28] Y. J. Cho, S. S. Dragomir, and Y.-H. Kim, "On some integral inequalities with iterated integrals," Journal of the Korean Mathematical Society, vol. 43, no. 3, pp. 563-578, 2006.

[29] W.-S. Wang, "A generalized sum-difference inequality and applications to partial difference equations," Advances in Difference Equations, vol. 2008, Article ID 695495, 12 pages, 2008.

[30] W.-S. Wang, "Estimation on certain nonlinear discrete inequality and applications to boundary value problem," Advances in Difference Equations, vol. 2009, Article ID 708587, 8 pages, 2009.

[31] K.-L. Zheng, S.-M. Zhong, and M. Ye, "Discrete nonlinear inequalities in time control systems," in Proceedings of the International Conference on Apperceiving Computing and Intelligence Analysis (ICACIA '09), pp. 403-406, October 2009.

[32] W.-S. Wang, Z. Li, and W.-S. Cheung, "Some new nonlinear retarded sum-difference inequalities with applications," Advances in Difference Equations, vol. 2011, no. 1, article 41, 2011.

[33] H. Zhou, D. Huang, W.-S. Wang, and J.-X. Xu, "Some new difference inequalities and an application to discrete-time control systems," Journal of Applied Mathematics, vol. 2012, Article ID 214609, 14 pages, 2012.

[34] Q. He, T. Sun, and H. Xi, "Dynamics of a family of nonlinear delay difference equations," Abstract and Applied Analysis, vol. 2013, Article ID 456530, 4 pages, 2013.

[35] Z.-X. Chen and K. H. Shon, "Fixed points of meromorphic solutions for some difference equations," Abstract and Applied Analysis, vol. 2013, Article ID 496096, 7 pages, 2013. 


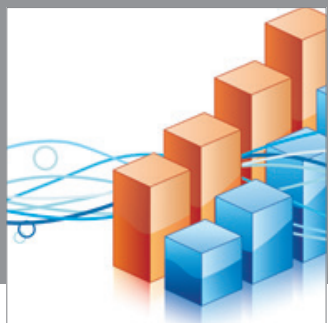

Advances in

Operations Research

mansans

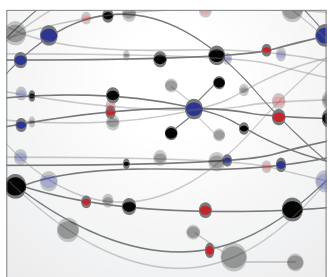

The Scientific World Journal
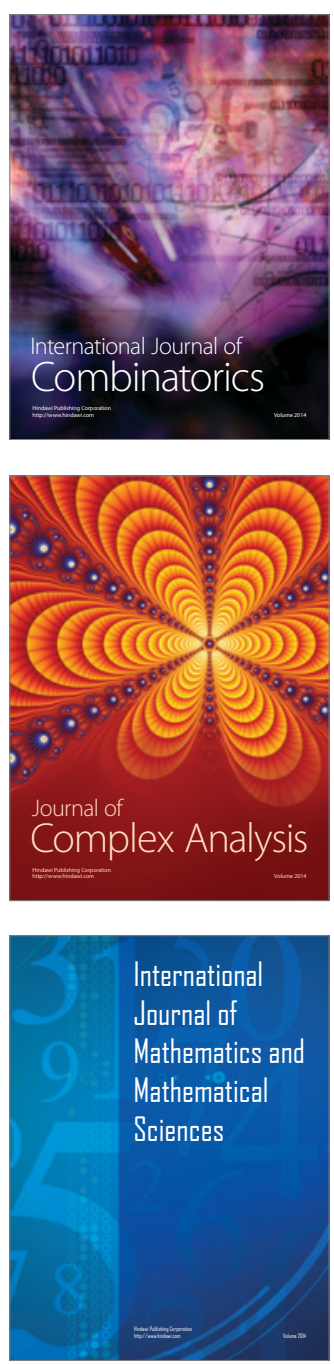
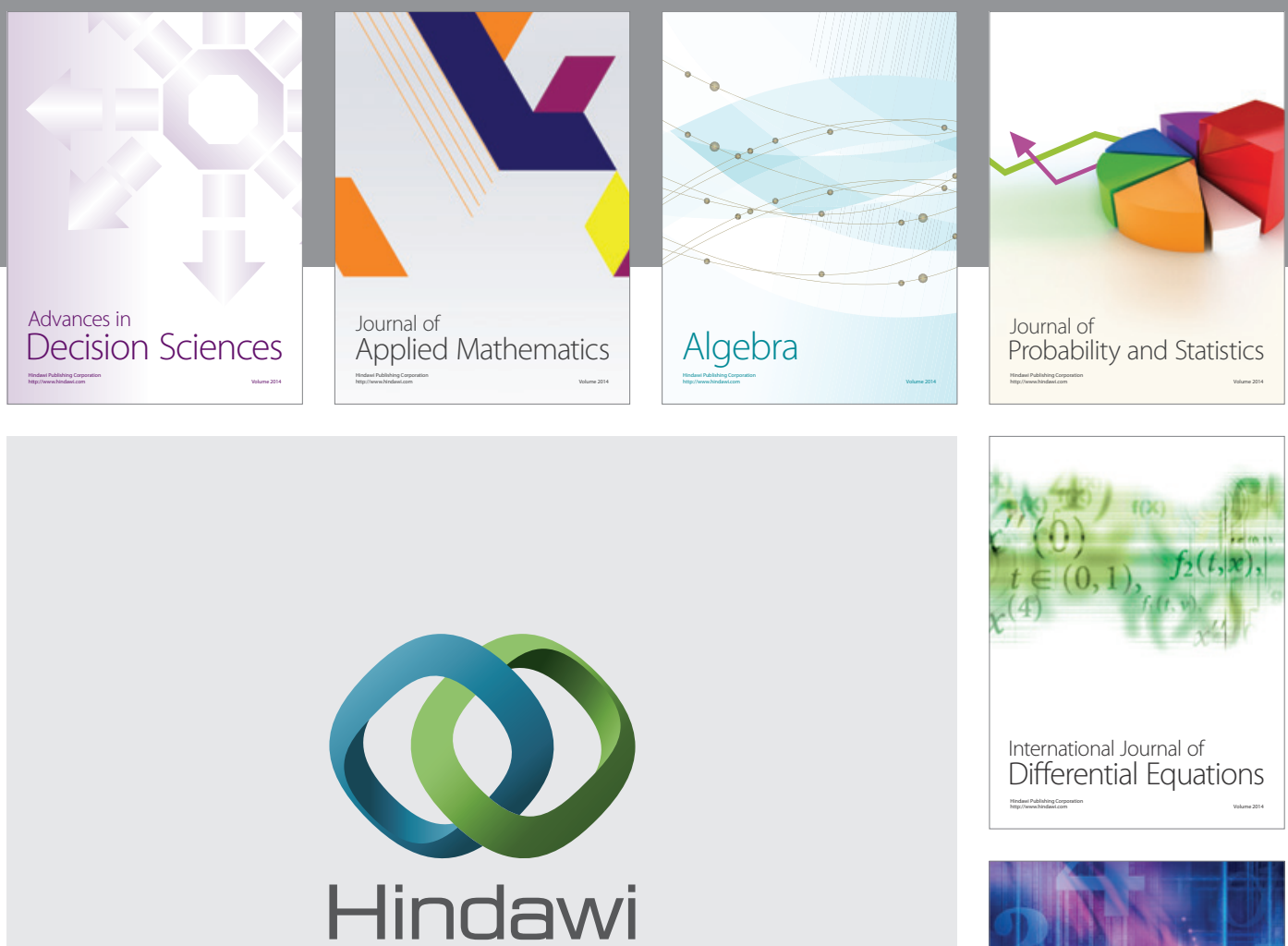

Submit your manuscripts at http://www.hindawi.com
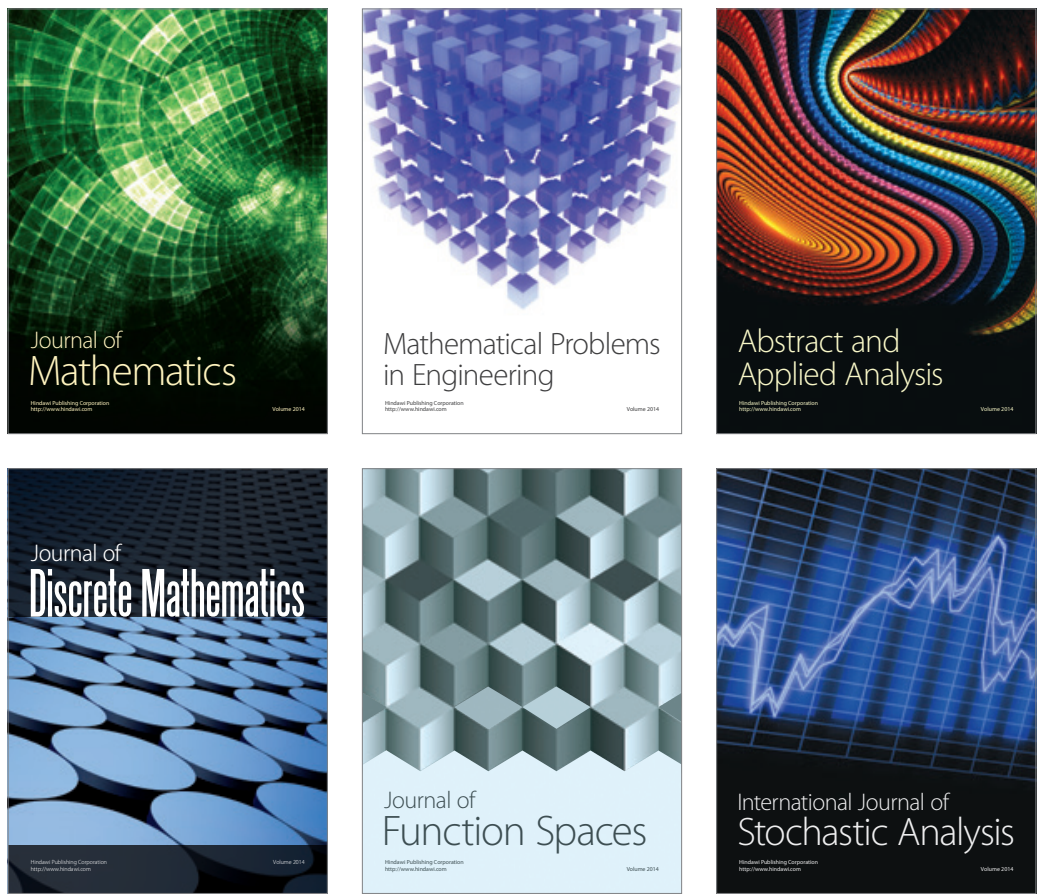

Journal of

Function Spaces

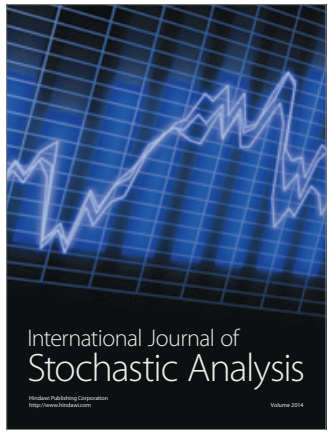

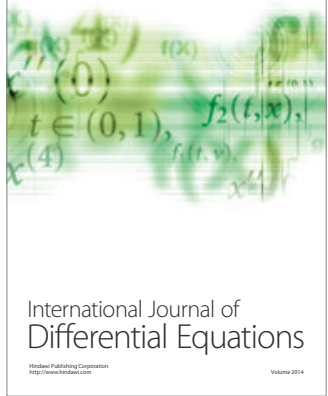
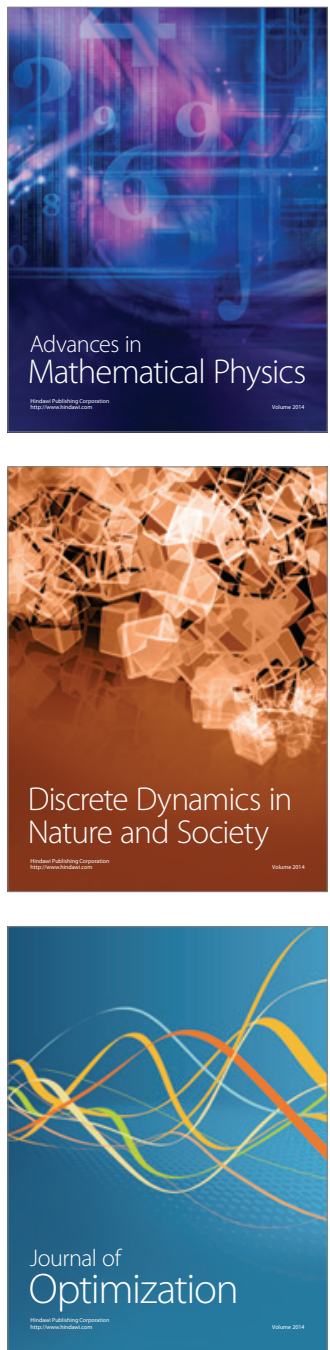